Comelli Cécilia, doctorante en géographie, UMR 5185 ADES-CNRS, université Bordeaux 3.

cecilia_comelli@hotmail.com

Masculin féminin : questions pour la géographie

\title{
Quelle incidence du genre sur la vie nocturne des étudiants bordelais?
}

"Grandes le jour, certaines villes se contractent en presque rien la nuit. Y auraitil deux villes lorsque nous prononçons le nom d'une seule? Ville-Jekyll et VilleHyde! " (Bureau, 2001).

\section{$\underline{\text { Introduction }}$}

Luc Gwiazdzinski écrivait en 2005 : "Si l'on connaît et l'on étudie depuis longtemps la ville diurne, on oublie encore souvent sa dimension nocturne. Privée de la moitié de son existence, comme amputée, la ville semble livrée aux seuls poètes et artistes noctambules." (Gwiazdzinski, 2005).Comme pour les études sur la temporalité nocturne de la ville, les études sur le genre, si elles sont fréquentes en sciences humaines et sociales en général, étaient relativement discrètes en géographie jusqu'à récemment. Il semble que la discipline ait désormais remédié à cet oubli.

Il m'est donc apparu pertinent de croiser ces deux objets de recherche que sont la nuit et le genre dans une étude de cas sur les pratiques et les représentations nocturnes des étudiant-e-s à Bordeaux. Dans cette étude, je me suis demandé si le genre de l'étudiant a une incidence sur sa manière de percevoir et de pratiquer la ville dans sa temporalité nocturne ? Sur quels aspects les deux sexes se retrouvent-ils ? Enfin, ces dernier-e-s ont-ils/elles des représentations et des territorialités propres à leur genre ? 


\section{I- Présentation de Bordeaux et de ses quartiers de vie nocturne}

\section{1- Bordeaux}

Importante ville universitaire, Bordeaux compte plus de 75000 étudiant-e-s dont plus de 60000 au sein des quatre universités de Bordeaux. Si les étudiant-e-s ne forment pas une classe sociale, ils représentent une véritable catégorie urbaine et des espaces sont définis par leur présence, notamment la place de la Victoire où ils sont nombreux à se retrouver dès le jeudi soir (E. Victoire, 2007). Néanmoins, Bordeaux n'est pas une ville réputée pour sa vie nocturne comme peuvent l'être Rennes, Montréal ou Barcelone. La vie nocturne bordelaise à évolué, et ce sont aujourd'hui principalement trois quartiers qui sont attractifs dans cette temporalité pour la population bordelaise. Les étudiant-e-s représentent une part importante des noctambules en étant des usagers nombreux et réguliers de cet espace-temps. Une étude réalisée auprès d'étudiant-e-s bordelais m'a permis de mettre en avant les perceptions et les pratiques nocturnes de cette population ${ }^{1}$. Si les données recueillies révèlent dans l'ensemble des approches comparables de la nuit par les deux sexes, ils se trouvent quelques aspects pour lesquels des nuances sont à préciser selon le genre des enquêtés. La principale différence constatée étant liée à la perception du danger et aux pratiques qui en découlent, ce qui nous amènera à nous pencher sur la place des femmes dans l'espace public la nuit.

Bordeaux est une ville qui a longtemps porté le surnom de "Belle endormie " par son manque de dynamisme, lié à un certain immobilisme et à une faible attractivité. La ville a subi de nombreuses mutations depuis 1995, date d'arrivée d'Alain Juppé à la mairie, ces mutations lui ont permis d'être classée au patrimoine mondial de l’Unesco en 2007.

Les principaux changements morphologiques sont l'arrivée du tramway, la mise en voies piétonnes d'un grand secteur du centre ville et les ravalements de façades. Cette évolution est aussi liée au phénomène de "retour en ville " que connaissent de nombreuses villes (Bidou-Zachariasen, 2003). Bordeaux ne fait pas exception en matière de rénovation et de marketing urbain. Les centres villes redeviennent attractifs et les quartiers ont tendance à se gentrifier. On y retrouve les mêmes politiques de rue piétonnes et les mêmes restructurations donnant de nouvelles fonctions aux lieux et transformant la vie économique de ces quartiers. (Roulleau-Berger, 2004).

Si plusieurs quartiers ou places de la ville ont une certaine activité nocturne, il en est trois dont l'activité est plus marquée (fig.1).

\footnotetext{
${ }^{1}$ Enquête statistique réalisée auprès de 1684 étudiants bordelais en 2010.
} 


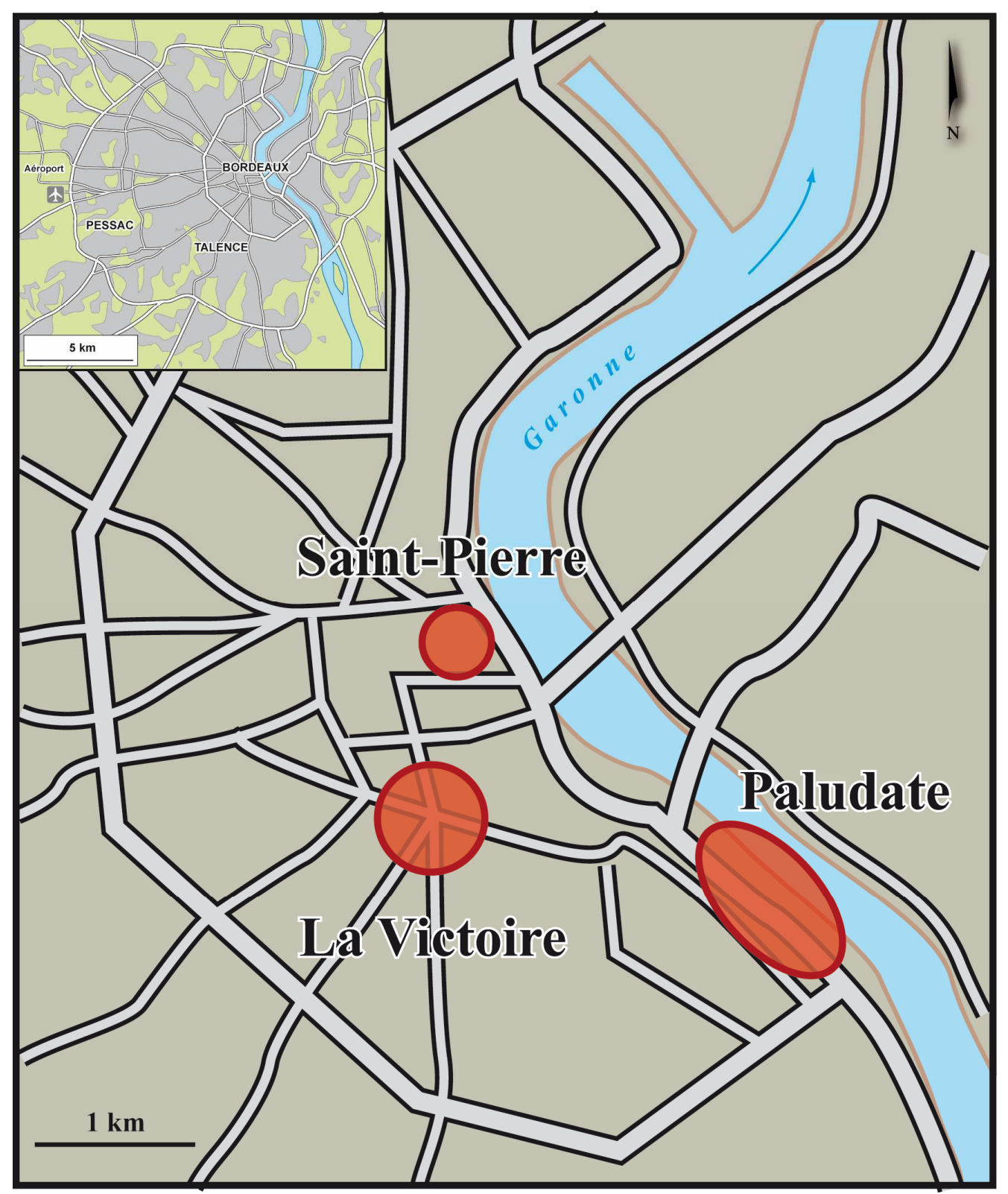

Fig.1 : Les principaux quartiers bordelais attractifs la nuit

\section{2- Les quartiers attractifs la nuit}

Un quartier en particulier a vu sa vie nocturne modifiée par ces changements, il s'agit du quartier St-Pierre, situé dans le centre historique de la ville. Ce quartier populaire avait une mauvaise réputation à la fin des années 80 , notamment du fait de la présence d'une population marginale de punks et de prostituées. S'il reste encore quelques travailleuses du sexe dans les coins un peu reculés, il s'agit désormais du quartier de prédilection des noctambules. Quartier " bobo " par excellence, on y retrouve le cinéma d'art et d'essai l'Utopia, des boutiques branchées-écolos, des bars à vin et surtout, plus de 150 restaurants et bars (fig.2). Ce quartier devenu piéton ne désemplit pas jusqu'à deux heures du matin; on y retrouve une importante population étudiante, les classes moyennes supérieures, et l'été une forte présence touristique. L'évolution de ce quartier central de 
Bordeaux est caractéristique d'une aseptisation et d'une homogénéisation croissante des centres villes. Martine Géronimi (2008) fait le même constat concernant le quartier ancien de la rue St-Paul à Montréal : ce dernier qui bénéficiait d'une mauvaise image il y a une vingtaine d'années est désormais un lieu festif, fort attractif pour les touristes avec des hôtels, des restaurants, des galeries d'art, en bref les rénovations et restructurations typiques qui conduisent à la gentrification des centres anciens.

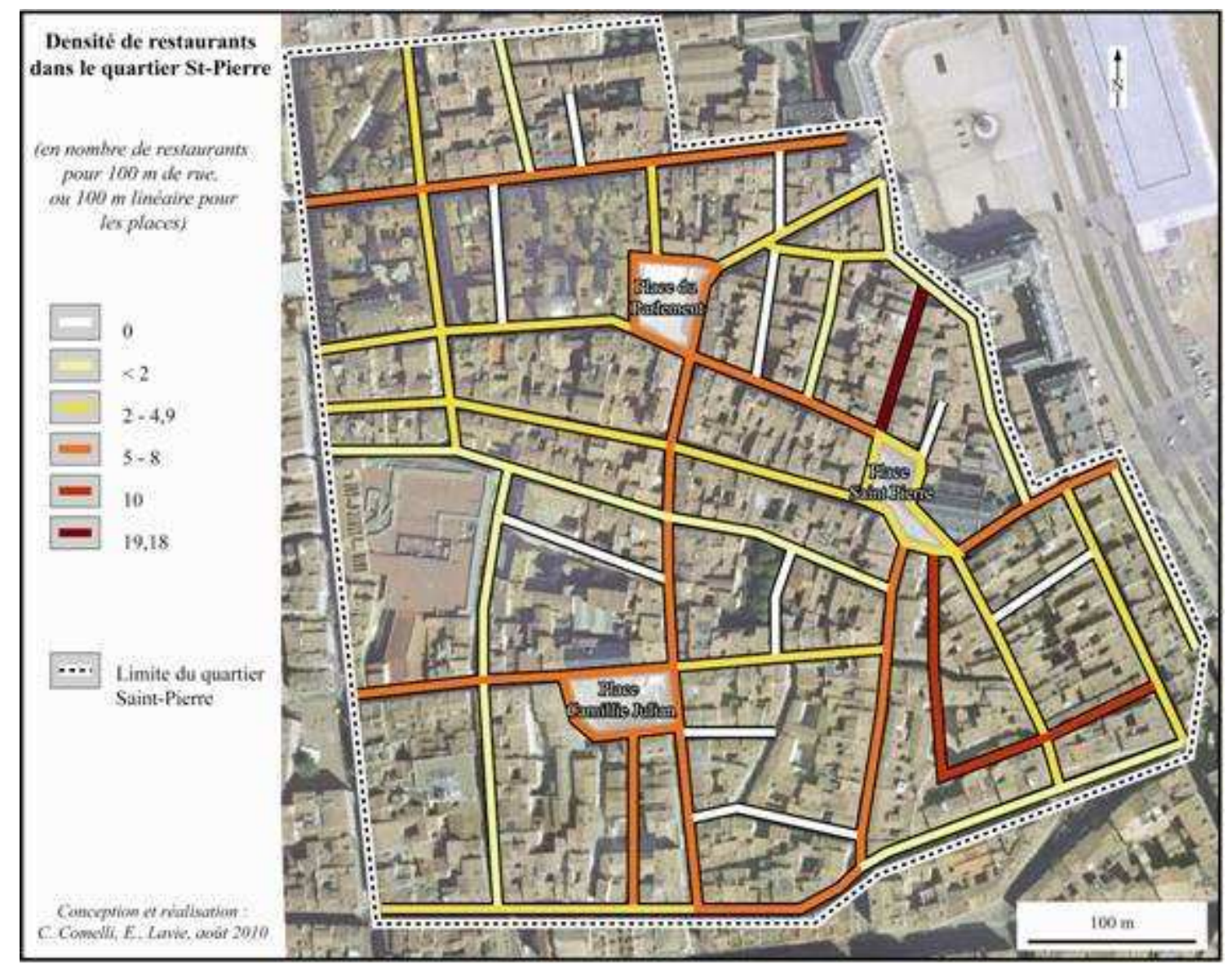

Fig.2 : La densité des restaurants à Saint Pierre

Un second quartier est historiquement le lieu de rendez-vous incontournable des étudiant-e-s, il s'agit de la place de la Victoire où là encore, des dizaines de bars se disputent la clientèle. Ce quartier très attractif reste encore populaire bien qu'il ait été en partie rénové depuis la mise en service du tramway. Dans leur ouvrage Sociologie de Bordeaux, les auteurs regroupés sous le nom d'Emile Victoire indiquent que cette place est " un lieu particulier: tous les jeudi soirs, elle est le théâtre d'une fête étudiante et populaire, fortement influencée par l'alcool, les traditions basques et les férias du sud-ouest [...]" (Victoire, 2007). Ce quartier est aussi l'un de ceux considéré comme dangereux par l'ensemble des étudiants.

Enfin, dernier lieu dans l'espace et dans le temps de la nuit bordelaise, les quais de Paludate (fig.3). Quartier reculé et moins " présentable " que le centreville, situé au bout des quais et derrière la gare, c'est loin d'être le secteur le 
plus rassurant de la ville. On pourrait même le qualifier de "glauque ". Il est marqué par la présence des entrepôts et des abattoirs. Il s'agit du lieu de concentration des discothèques de la ville depuis les années 1990; on en dénombre une vingtaine pour tous les goûts : de l'ambiance généraliste à la musique africaine, en passant par le jazz et sans oublier la boîte "gay ". La mise en lumière par la municipalité faisant défaut, ce sont les néons des discothèques qui prennent le relais pour éclairer leur pas de porte, de plus il s'agit d'un des principaux secteurs de la prostitution à Bordeaux. Les habitants se sentent mis à l'écart par le manque d'attention portée à ce quartier par rapport au reste de la ville par les autorités. Le sentiment d’insécurité y est fort (E. Victoire, 2007), aussi bien parmi les résidents que parmi les noctambules fréquentant le site.

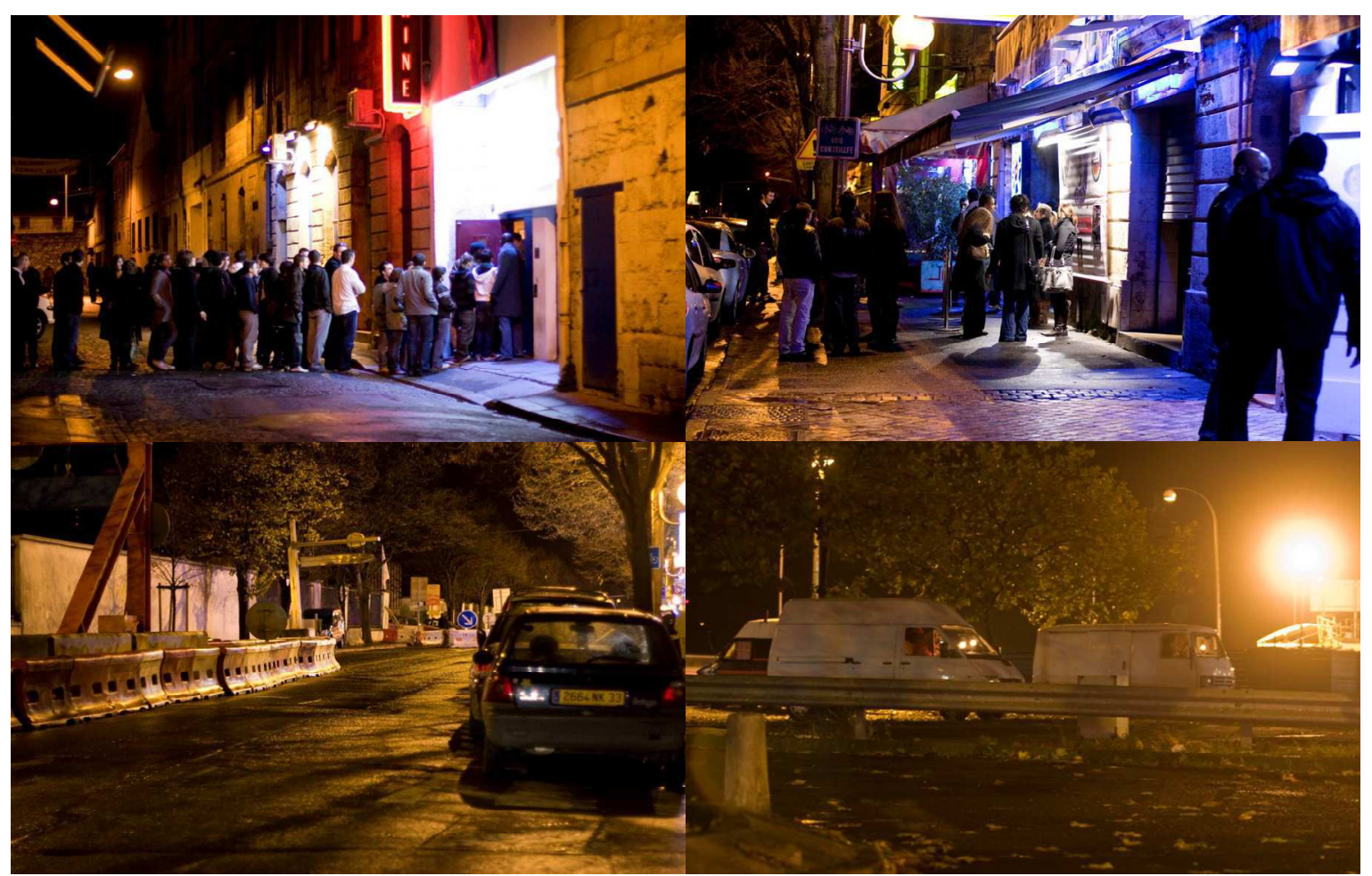

Fig.3 : Le quai de Paludate. Photos : Julie Cazzulo, DR

En le pratiquant, on ne peut que remarquer le peu d'attention portée à l'aménagement et à la mise en lumière de ce secteur pourtant attractif la nuit, à la différence de la place de la Bourse par exemple située un peu plus au nord des quais. Ce qui nous renvoie au propos de Luc Bureau dans son ouvrage Géographie de la nuit (1997) : pour lui, la lumière du soleil est cartésienne, elle ne privilégie aucun point de l'espace, alors que la lumière qui scintille la nuit est du fait de l'homme, lequel éclaire en principe ce qui vaut la peine de l'être. Ce quartier attractif mais peu rassurant et ses noctambules ne mériteraient donc pas cette attention. 
Après un tour d'horizon de la ville de Bordeaux et de ses quartiers attractifs la nuit, qu'en est-il des pratiques estudiantines ?

\section{II- Les résultats de l'enquête : quelle est l'incidence du genre dans les rapports des étudiant-e-s à la nuit ?}

Pour la plupart des données recueillies on observe peu de différence entre les sexes et quand il y en a, on n'est pas loin des stéréotypes. Pour tous : "La nuit est un temps différent où les valeurs ont tendance à s'inverser, où les désirs et les peurs s'exacerbent. Pour ceux qui sortent de nuit, la multi-activité est de mise: travail, loisirs, rencontres, balades nocturnes, activités culturelles ou artistiques, fréquentations des bars ou des boîtes, mais aussi consommation d'alcool et de drogues. " (Moutchouris, 2003). Ces éléments se retrouvent dans l'enquête menée auprès de la population étudiante bordelaise.

\section{1- Les heures de la nuit :}

Les étudiants interrogés ont une même idée des bornes horaires de la nuit: quel que soit le sexe, la nuit débute à $21 \mathrm{~h}$ pour environ $30 \%$ et à $22 \mathrm{~h}$ pour 33\% d'entre eux. Elle se termine à $6 \mathrm{~h}$ pour plus de $40 \%$ des étudiants (fig.4). Gwiazdzinski, quant à lui dans ses recherches, borne la nuit entre $20 \mathrm{~h}$ et $6 h$.

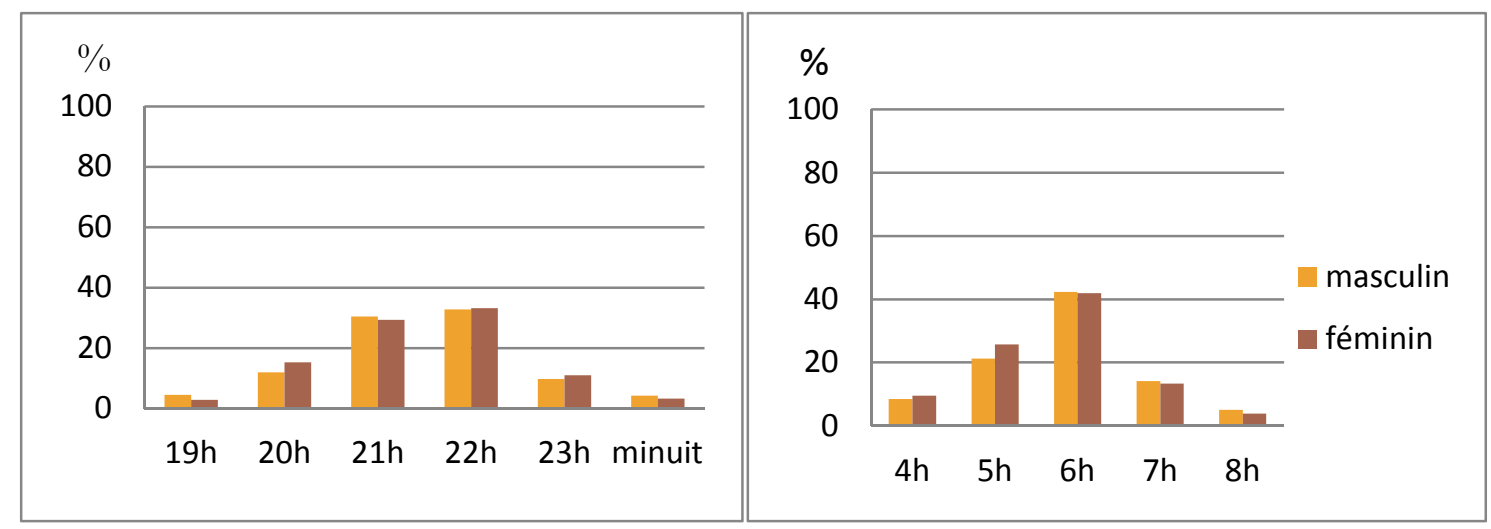

Fig.4 : Les bornes horaires de la nuit selon les étudiant-e-s

Si les étudiant-e-s sortent de chez eux en moyenne entre $20 \mathrm{~h}$ et $22 \mathrm{~h}$; la plupart entre $20 \mathrm{~h}$ et $21 \mathrm{~h}$ (40\%), et environ $30 \%$ entre $21 \mathrm{~h}$ et $22 \mathrm{~h}$ (fig.5), et s'ils ont une fréquence de sortie similaire (30 à 40\% sortent une à deux fois par semaine), une première petite différence apparait concernant l'heure de retour chez soi : les étudiantes rentrent en moyenne un peu plus tôt que les étudiants, environ $40 \%$ de ces dernières rentrent entre minuit et $2 \mathrm{~h}$ du matin, 
quand la même proportion d'étudiants déclarent rentrer entre 2 et $4 \mathrm{~h}$ du matin.
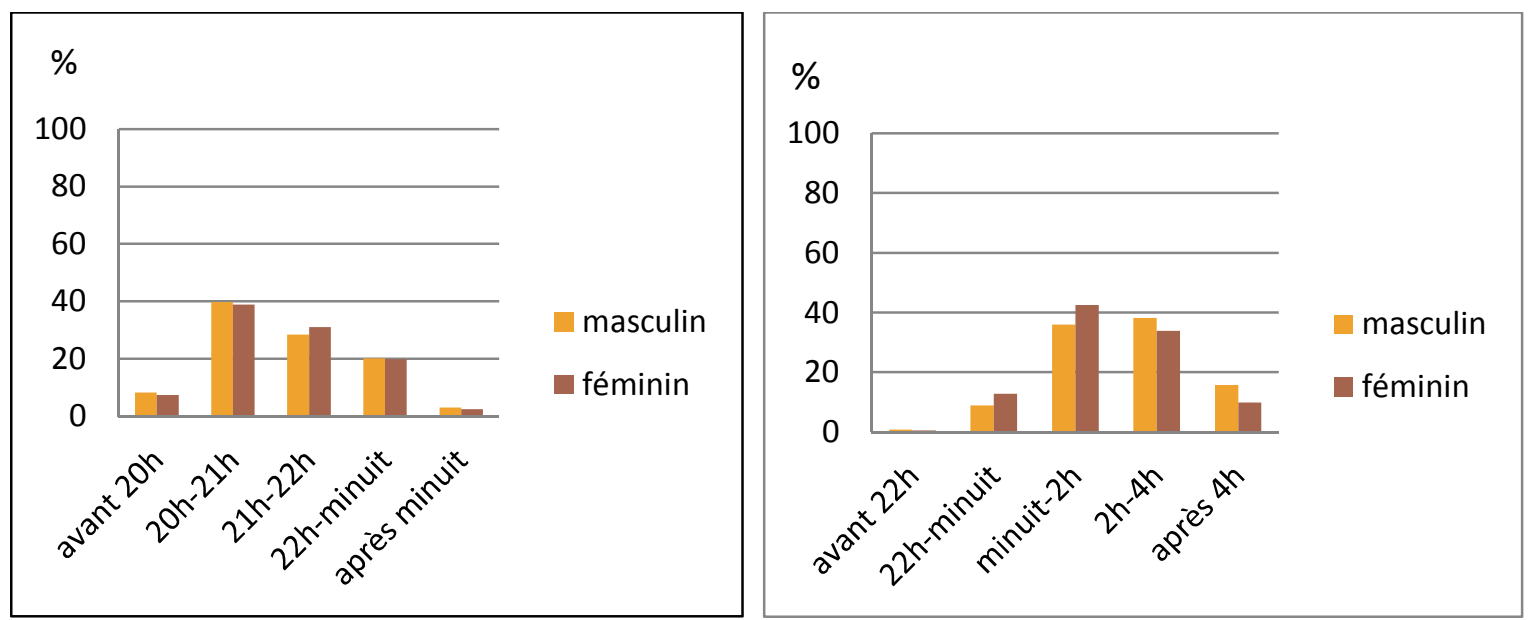

Fig. 5 : Les heures auxquelles les étudiant-e-s sortent de chez eux

\section{2- Les représentations de la nuit par les étudiant-e-s}

Quand il est demandé aux étudiant-e-s ce que leur évoque la nuit, les mêmes thèmes dans le même ordre reviennent au premier mot donné. La réponse donnée par le plus grand nombre est une série de mot en rapport avec l'absence de lumière (noire, obscure, sombre, etc.), viennent ensuite les champs lexicaux de la fête (environ 20\%), puis du sommeil (15\%), ensuite du calme, de la convivialité et enfin, celui de l'alcool (fig.6).

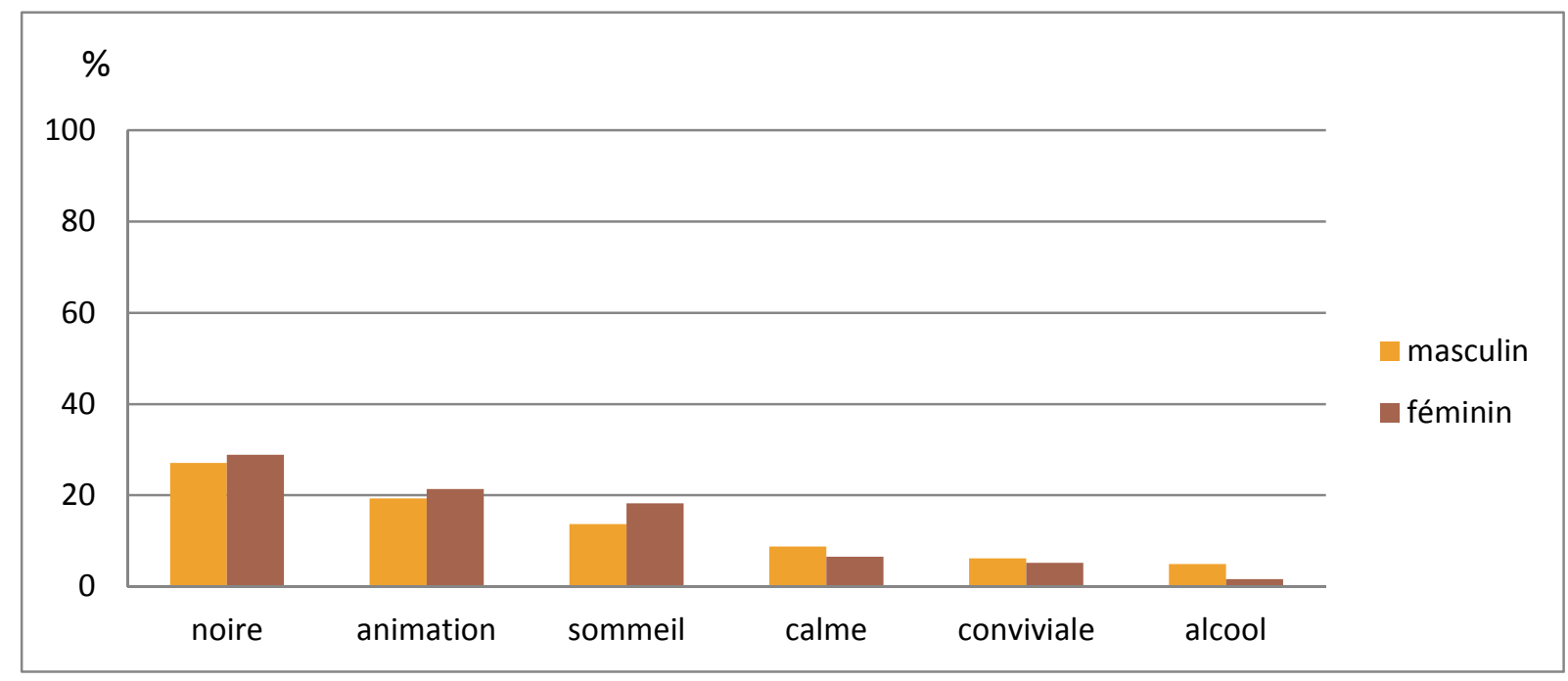

Fig. 5 : Les mots évoquant la nuit selon les étudiant-e-s 
Les champs lexicaux évoqués sont les mêmes par les deux sexes mais l'on peut constater un léger écart dans les proportions : les filles font plus souvent référence au sommeil et les garçons à l'alcool.

\section{3- Les motivations poussant les étudiant-e-s à sortir}

A la question "pour quelles raisons sortez-vous?", ce sont les mêmes motivations qui apparaissent au premier abord. "Voir des amis ", "se promener ", " boire " et " danser ". Ces résultat nous rappellent la vision d'Emile Durkheim, selon laquelle "Le trait caractéristique de la fête \{...] est d'être un rassemblement de masse, générateur d'exaltation, plein de cri, de chants, de danses, de tumulte et d'ivresse." (Durkheim, 1912). Dans son étude sur les jeunes de la nuit, Antigone Moutchouris fait un constat similaire, la nuit : " [...] c'est un temps de compensation, de recréation [...]. C'est un temps de convivialité, de rencontres, de sensualité, de fêtes, de transgression." (Moutchouris, 2003).

En observant les résultats de plus près, on remarque que les proportions pour chaque réponse donnée varient plus ou moins selon le sexe. La principale motivation pour tous est de sortir pour "voir des amis ", mais les filles sont un peu plus nombreuses à le dire $(80 \%$ des étudiantes et $70 \%$ des étudiants). Les différences sont plus marquantes sur d'autres points : 34\% des étudiantes sortent pour danser contre à peine 20\% des étudiants, 38\% de ces derniers déclarent sortir pour boire ce qui concerne $27 \%$ des étudiantes, ils sont même près de $11 \%$ à déclarer sortir le soir pour se saouler, moins de $4 \%$ des étudiantes ont donné cette réponse. Les garçons sont plus nombreux sur un dernier point: ils sont $18 \%$ à sortir dans le but (l'espoir ?) de faire des rencontres amoureuses, c'est une des raisons données par seulement $5 \%$ des étudiantes. Le sexe et l'alcool seraient donc l'apanage des garçons (fig.7).

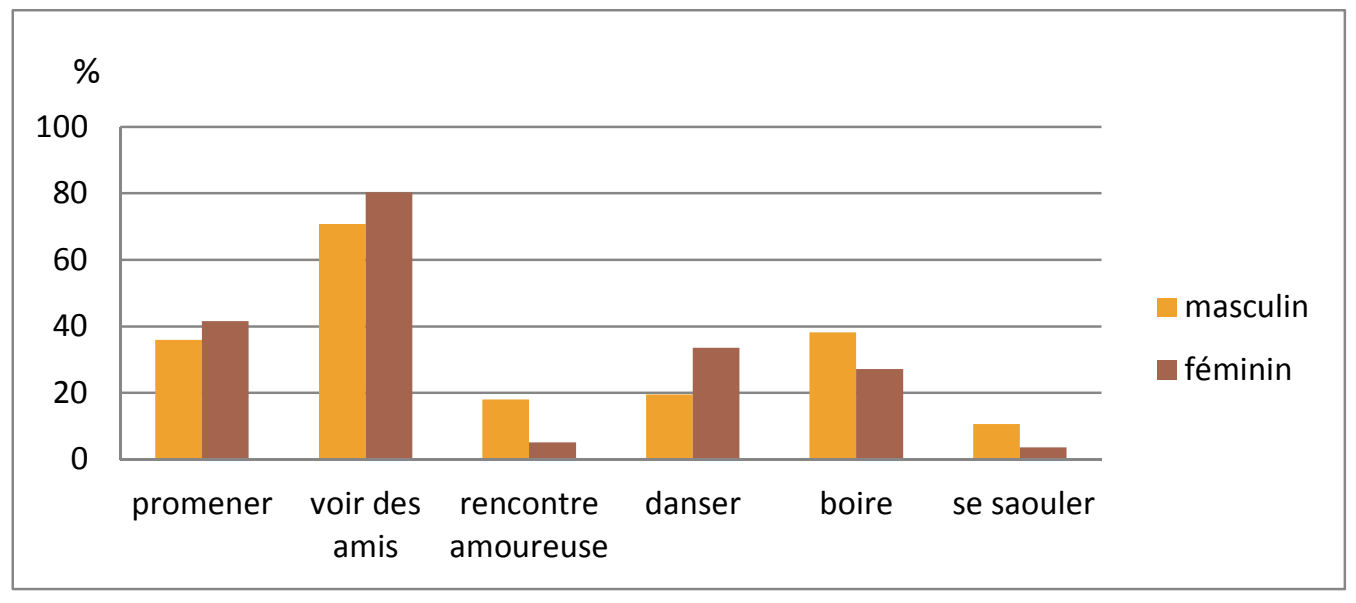

Fig.7 : Les principales motivations des étudiant-e-s pour sortir le soir

\section{4- Que consomment les étudiant-e-s lorsqu'ils/elles sortent ?}


Les principales consommations des étudiants en soirée sont l'alcool et le cannabis. Autour de $65 \%$ des étudiant-e-s déclarent consommer de la bière ou du vin et $55 \%$ des alcools forts, Pour Luc Gwiazdzinski : "Cette conquête de la nuit est rendue possible par la consommation d'excitants, comme le café, les cigarettes ou les vitamines." (Gwiazdzinski, 2005).

D'après les statistiques nationales, les filles consommeraient un peu moins d'alcool que les garçons. Si l'on regarde les moyens de transports utilisés, elles sont un peu plus nombreuses à utiliser leur voiture, on peut donc penser qu'elles consomment effectivement moins d'alcool en quantité même si elles sont autant à déclarer en boire. En revanche, leur consommation déclarée de stupéfiants est inferieure à celle des étudiants. Elles seraient presque $14 \%$ à consommer du cannabis ou de l'herbe pour presque $25 \%$ des étudiants. Si le pourcentage d'étudiants des deux sexes déclarant prendre de l'ecstasy ou de la cocaïne est relativement faible, il reste plus élevé chez les garçons, deux fois plus déclarent prendre des ecstasy et presque quatre fois plus de la cocaïne. Près de $10 \%$ des étudiants ne consomme ni alcool, ni drogue (fig.8).

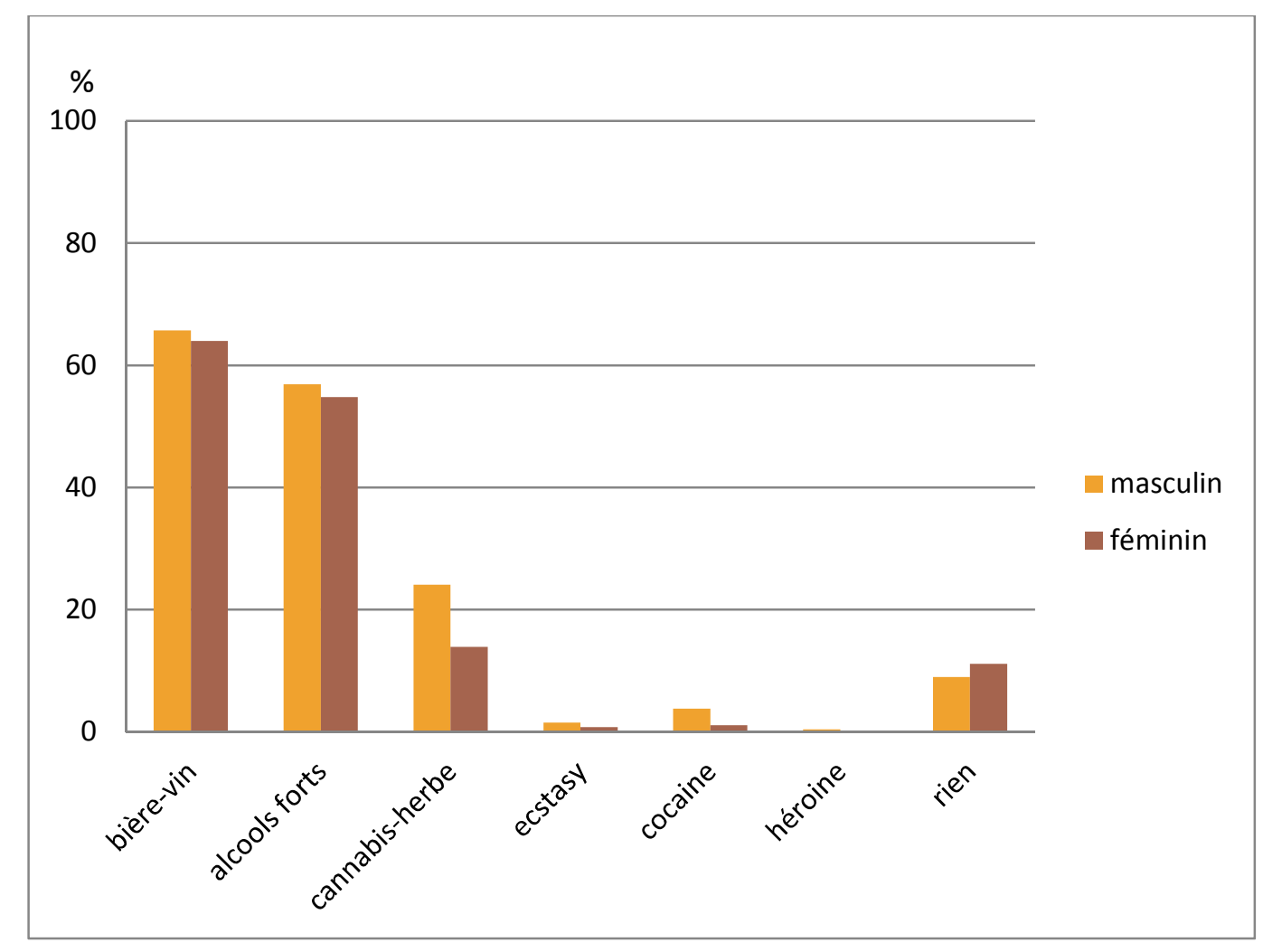

Fig.8 : les produits consommés en soirée par les étudiant-e-s

\section{5- Les préparatifs : la césure entre le jour et la nuit}

La préparation avant de sortir est un temps important de la soirée, pour J.M Deleuil (1994), la césure entre le jour et la nuit est marquée, quelle que soit l'heure, par les préparatifs des individus qui se "parent " pour sortir. Cette pratique concerne davantage les étudiantes. Elles sont plus nombreuses à se 
préparer et y consacrent plus de temps. Les trois quarts se préparent contre la moitié des étudiants. Si 16\% des étudiants prennent moins de 5 minutes pour ces préparatifs, elles ne sont que $4 \%$ à utiliser si peu de temps; à l'inverse, elles sont près de $24 \%$ à passer entre 30 minutes et 1 heure à se préparer pour $8,5 \%$ des étudiants. La majorité des étudiantes va consacrer de 15 à 30 minutes à ces préparatifs, les étudiants y consacrent plus fréquemment entre 5 et 15 minutes (fig.9). Comme le note Thierry Paquot : "La ville se fait volontiers cabine d'essayage et tremplin de défilés de mode improvisés, les corps y évoluent comme ceux des mannequins." (Paquot, 2006).

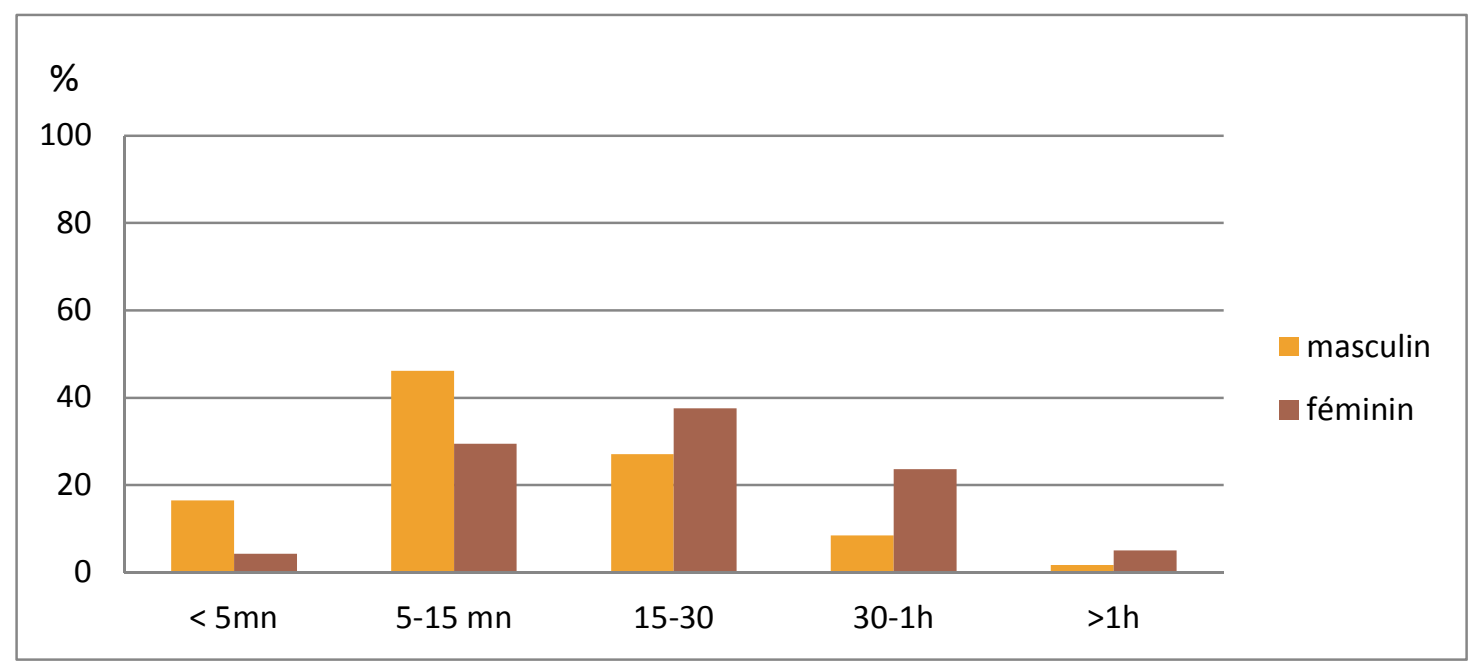

Fig.9 : Le temps consacré par les étudiant-e-s à la préparation avant de sortir

\section{6- Danger, attraction, répulsion : une appréhension différente de la ville et de ses quartiers}

Les étudiant-e-s ont aussi été interrogés sur la "dangerosité " perçue de la nuit. La peur de la nuit est ancestrale, la peur du non retour du jour et de la présence démoniaque hante les esprits, aidée en ce sens, durant une longue période, par l'Eglise qui diffuse et encourage cette image maléfique (Delattre, 2000). Cette crainte persistera malgré les découvertes astronomiques. Déjà en 1654 Gassendi écrivait : "La succession des nuits et des jours n'existe que d'un point de vue relatif, pour un observateur placé sur la terre; le concept de nuit n'a aucun sens sans l'immensité de l'univers, la terre n'est pas le centre de l'univers, mais uniquement un point privilégié, car celui de l'observateur ". Il savait qu'il n'y avait pas de raison de s'inquiéter de l'absence du jour. Au-delà de l'aspect astronomique, ce qui inquiète encore de nos jours dans cette obscurité, c'est justement le fait de ne pas voir le danger arriver, or, la vue est le sens que nous utilisons le plus. La nuit, la rue est donc tout à la fois attractive et répulsive. Cet imaginaire est fortement lié à la présence de l'éclairage public (Géronimi, 2008). Dans le cadre de l'enquête, une grande majorité des étudiant-e-s a déclaré ne pas trouver la nuit dangereuse, néanmoins, dans leurs pratiques 
respectives, des différences sont bien repérables en particulier quant aux quartiers attractifs ou répulsifs selon le sexe.

Sur l'aspect dangereux de la nuit, les étudiantes sont plus nombreuses à penser qu'elle l'est: elles sont 30\% à le dire contre $20 \%$ des étudiants. Bordeaux n'est pas de manière générale considérée comme une ville dangereuse, les faits divers relatés dans le journal Sud-Ouest sont peu nombreux et ne concernent pas fréquemment les agressions physiques. D'après le responsable de la police municipale, parmi les agglomérations françaises de tailles similaires à Bordeaux, cette dernière est celle où le taux de délinquance est le plus faible. La perception du danger est donc assez éloignée de la réalité des faits. En effet, quand on questionne les étudiant-e-s sur la dangerosité plus localisée de Bordeaux la nuit, les filles sont presque $80 \%$ à trouver qu'il existe des quartiers dangereux, ce qui est le cas pour un peu moins de 60\% des garçons (fig.10), elles sont de ce fait bien plus nombreuses à déclarer sortir en groupe par crainte, près de 40\% d'entre-elles contre 9\% des étudiants (fig. 11).

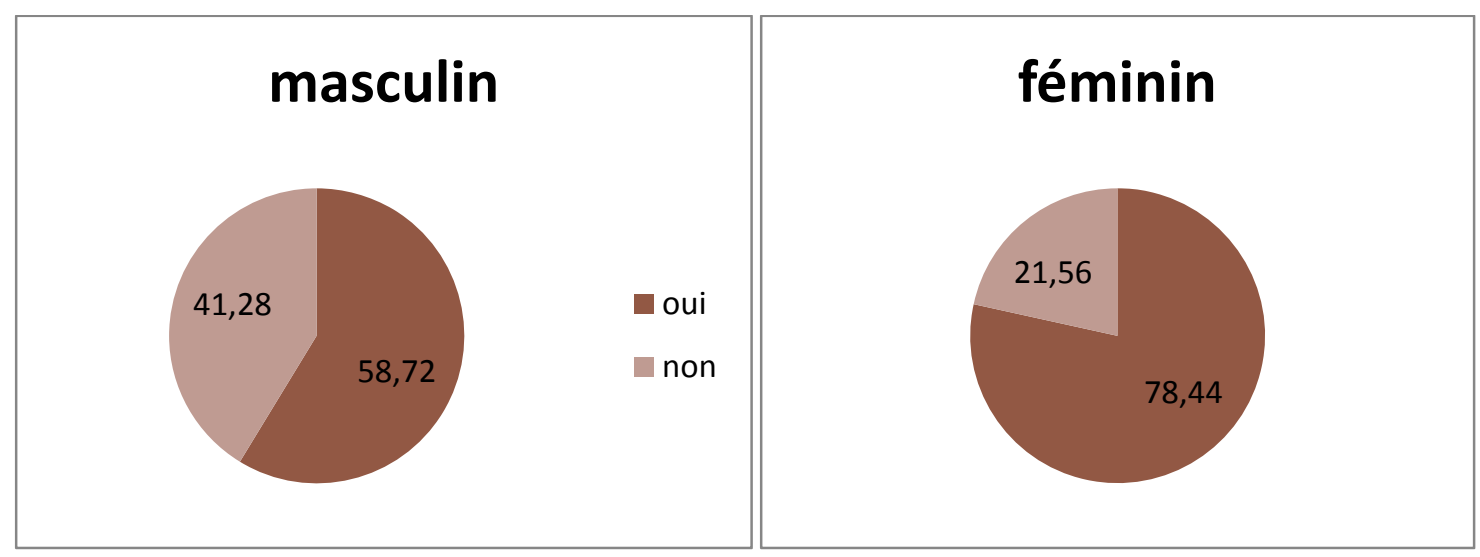

Fig.10 : Les étudiant-e-s pour lesquel(le)s il existe des quartiers dangereux la nuit à Bordeaux

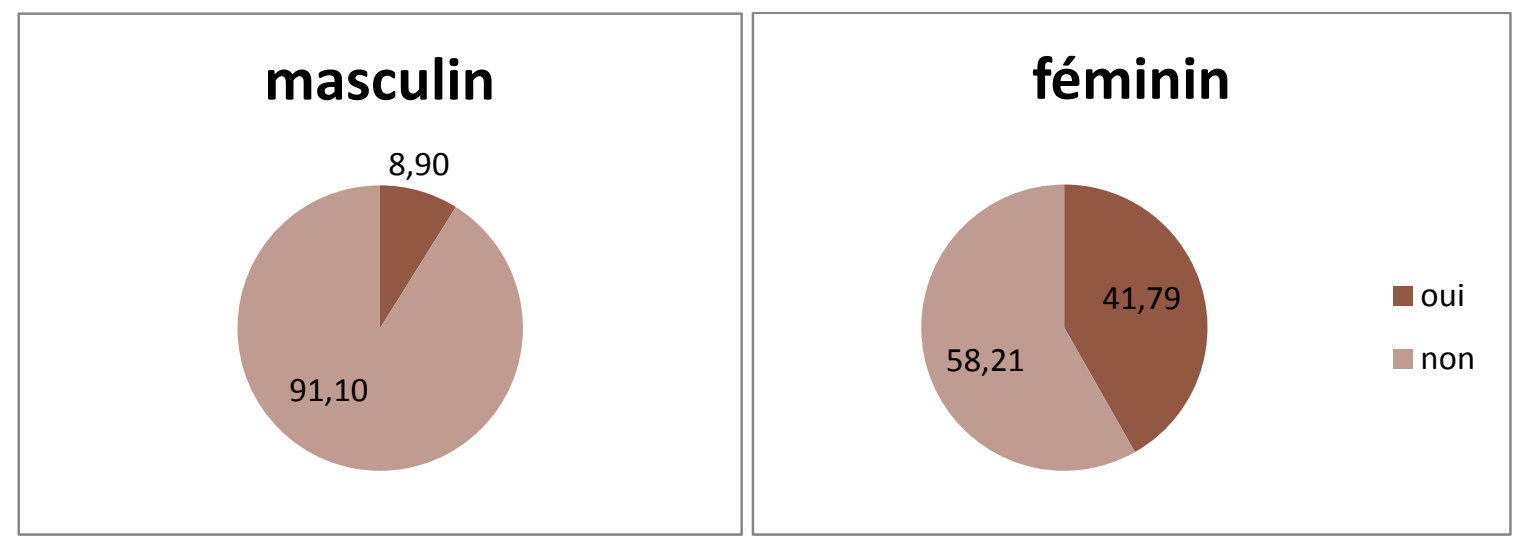

Fig.11 : Les étudiant-e-s sortant en groupe par crainte 
Les quartiers dangereux sont les mêmes cités par les deux sexes : il s'agit de la gare pour près de $50 \%$ des étudiants, de la Victoire pour près de $40 \%$ et de St-Michel à plus de 35\%, ce sont des quartiers majoritairement populaires. Dans les études réalisées sur la violence urbaine, il apparaît clairement un lien entre les quartiers supposés dangereux et la population d'origine immigrée dans le discours des enquêtés, c'est le cas pour les quartiers cités par les étudiant-e-s (Lieber, 2008). Certain-e-s ont aussi dit craindre les cités, précisant parfois qu'ils/elles n'y sont jamais allé-e-s. Néanmoins, les jeunes qui sortent plus fréquemment la nuit ont moins d'appréhensions que ceux qui en sont moins familiers, ils ont une meilleure appropriation de cet espace-temps (Moutchouris, 2003).

Les mêmes quartiers sont considérés comme attractifs pour les étudiante-s. Les deux quartiers les plus fréquentés sont ceux de St-Pierre et de la Victoire. Si le quartier de la Victoire est considéré comme le plus dangereux pour environ $40 \%$ des étudiant-e-s ayant répondu à la question, c'est celui qui est le plus fréquenté par les étudiants (à près de 44\%) alors que moins d'un tiers des étudiantes déclarent y sortir, ces dernières privilégiant le quartier StPierre à 60\% (fig.12).

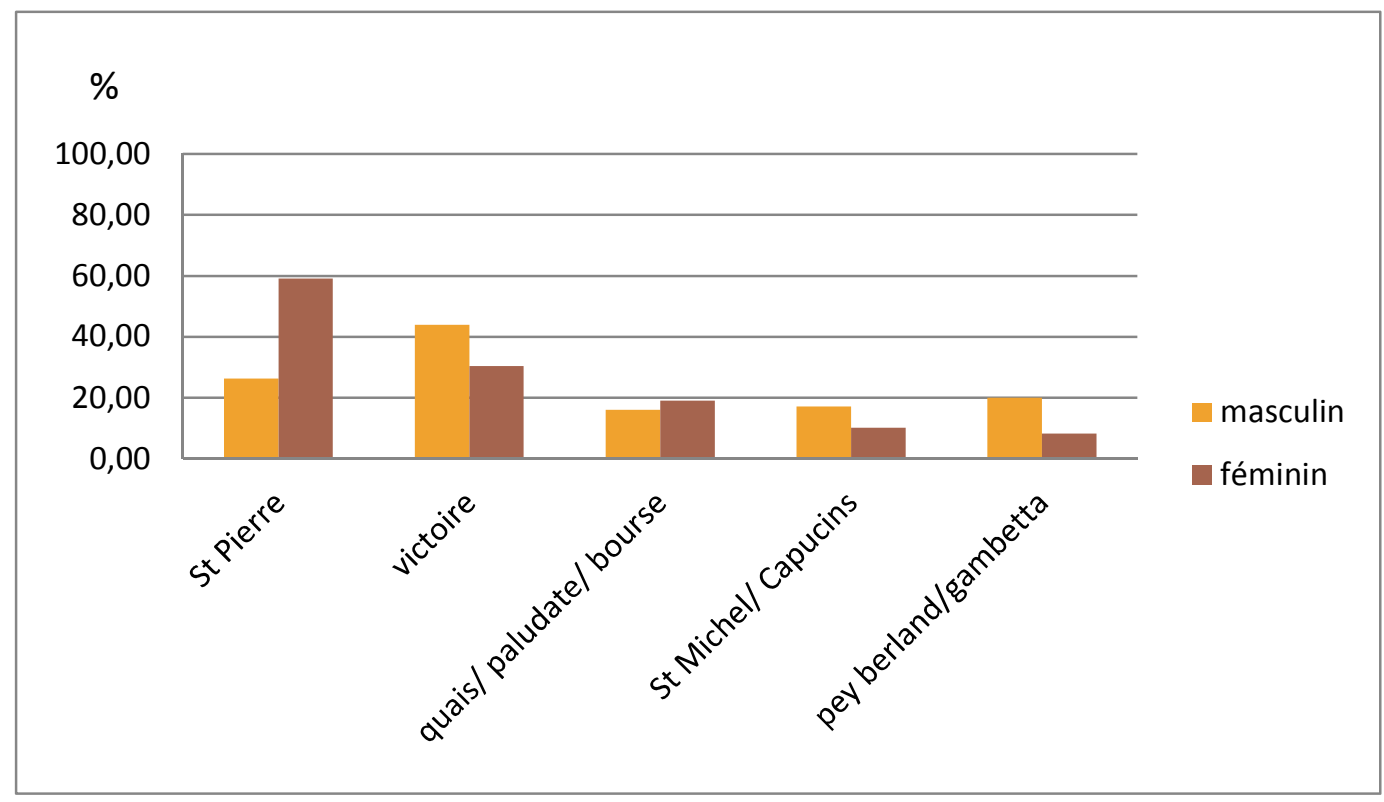

Fig.12 : Les quartiers privilégiés de sorties pour les étudiant-e-s

La différence la plus marquante entre les sexes concerne donc la notion de danger et en particulier les représentations et les pratiques qu'elle induit chez les étudiantes.

\section{III- $\quad$ Rendre la ville accessible aux femmes la nuit}




\section{1- La vulnérabilité des femmes, un principe intégré dès le plus jeune âge}

Toutes les études réalisées sur les violences et les femmes dans l'espace public en arrivent à la conclusion que la rue, en particulier la nuit, est un territoire qui appartient majoritairement aux hommes et que les femmes pratiquent avec une certaine réticence. On peut citer le constat fait, entre autre, Laurence Roulleau-Berger pour qui : "Aujourd'hui, si les hommes et les femmes [...] peuvent s'exposer sur un mode paritaire dans la rue, il semblerait cependant que la rue contienne des dissymétries sociales (Gautrat, 1996). Par exemple, la nuit, la rue reste inhospitalière pour les femmes. Elles ne la perçoivent pas comme accessible, elles y circulent moins nombreuses que les hommes et, de toutes façons, ne peuvent s'y arrêter: Il y a toujours la peur d'être accostée, suivie, agressée. [...]. Il y a toujours dans les sociétés européennes un consensus implicite autour de l'idée que la rue, la nuit, n'appartient pas aux femmes [...]. " (Roulleau-Berger 2004). Cette idée de danger est supérieure à la réalité statistique des agressions envers les femmes, cependant les chiffres ne retiennent que les agressions physiques, et ne prennent pas en compte les agressions verbales ou le fait d'être " simplement "importunée par les hommes. Or, comme le constate Marylène Lieber (2008), ces intrusions des hommes dans la sphère privée des femmes sont souvent vécues par ces dernières comme une agression, un rapport social qui n'est pas forcement désiré et qui s'en trouve déplacé. Ce sont les multiples remarques, pas toujours négatives, qui rendent les femmes plus anxieuses dans la rue la nuit, car elles savent qu'elles risquent d'être importunées sans savoir jusqu'où va aller l'interlocuteur, du simple compliment à l'agression sexuelle. Les hommes quant à eux craignent plus de se faire dérober leurs biens que pour leur personne.

Sortir pour une femme la nuit, dans la plupart des cas, n'est donc pas anodin; à la différence des hommes, elles anticipent ce qu'elles vont porter selon qu'elles sont seules ou accompagnées, quel trajet elles vont emprunter, quel mode de transport elles vont utiliser. Cela fait partie des stratégies mises en place par les femmes quand elles sortent. Ne pas rentrer seule, ne pas porter de tenue " provocante ", ne pas croiser le regard des hommes pour éviter qu'ils prennent cela comme une invitation, éviter certaines rues ou encore porter des chaussures qui permettent de courir si nécessaire. Cela se fait souvent de manière naturelle, voire inconsciente, car ces appréhensions sont validées par l'éducation des filles dès le plus jeune âge. À l'adolescence par exemple, elles ont moins de liberté que les garçons, les parents s'inquiètent plus de leurs fréquentations, des lieux de sortie et ils sont plus stricts sur les horaires. Elles intègrent donc très jeunes le fait que la rue est un danger pour elles, elles sont conditionnées pour avoir peur la nuit. Ce sentiment est aussi renforcé par les médias (Lieber, 2008). Les étudiantes enquêtées rapportent très souvent qu'elles font plus attention la nuit et qu'elles essaient de contourner les risques en évitant d'être seule ou en prenant un taxi. Les femmes sont considérées comme étant vulnérables la nuit de par leur 
" faiblesse " physique, mais aussi par un apprentissage tout au long de la vie $\mathrm{du}$ comportement socialement attendu de leur part. Les différences entre les sexes sont donc avant tout le produit d'une construction sociale (Bereni, Chauvin, Jaunait, Revillard, 2008).

On a vu que la crainte de certains quartiers a une incidence sur les pratiques territoriales, en particulier des filles, puisqu'elles pratiquent bien plus fortement St-Pierre que la Victoire, ce secteur étant considéré comme le quartier le plus dangereux après la gare. Cela va dans le sens des propos de Martine Géronimi sur l'exemple Montréalais pour expliquer ces pratiques différentes. En effet, elle nous apprend dans son article que la rue St-Paul est désormais la nuit une rue éclairée avec goût pour mettre en évidence l'esthétique des bâtiments victoriens et le caractère bourgeois et de bon aloi du paysage, ce qui permet aux usagers de profiter de la nuit paisible dans un lieu festif sans crainte. Si le quartier Saint Pierre n'est pas vraiment qualifiable de " paisible ", sa population nocturne y étant plus jeune que celle de la rue Saint Paul à Montréal et les conflits d'usages y étant assez fréquents avec les habitants du quartier qui aspirent à plus de calme, il n'est pas considéré comme dangereux car on y retrouve le même schéma de quartier rénové, mis en lumière et ainsi rassurant et pratiqué par deux tiers des étudiantes.

Il est intéressant de constater que les filles fréquentent un peu plus les quais que les garçons alors que c'est l'un des quartiers considérés comme dangereux. On peut expliquer cela car les étudiantes sont plus nombreuses à déclarer sortir pour danser, or, Paludate est le lieu par excellence pour cette pratique. Nous avons aussi vu précédemment que les filles déclarent éviter de sortir seule dans certains quartiers ou à certaines heures. Elles ne se privent pas d'aller danser, mais adoptent une stratégie de groupe pour s'y rendre. On pourrait parler de " performance " de la part des étudiantes dans leur conquête de la ville nocturne.

\section{2- La gentrification : la solution pour une ville plus rassurante ?}

Si la nuit n'est pas sereine pour beaucoup de femmes, la gentrification, malgré les discriminations dont elle est responsable, rend possible une ville nocturne sans crainte dans certains quartiers, ce que nous montre l'exemple des étudiantes sortant majoritairement à St-Pierre.

L'évolution de la gentrification signifie-t-elle pour autant la mort nocturne des quartiers en marge des aménagements ? Cette situation peut nous mettre dans une position contradictoire. D'une part, on peut s'opposer à l'homogénéisation et craindre que cette tendance à l'aseptisation et au beau ne se développe à l'ensemble de la ville, mettant fin, dans le cas de Paludate, à la vie nocturne de ce secteur. Mais d'autre part, on ne peut que vouloir qu'une plus grande attention soit portée à ce secteur, au moins en matière de mise en 
lumière, pour le rendre plus attractif pour une population plus diversifiée mais aussi pour les femmes.

Ce dernier n'est pas absent des aménagements sans raison, il faut tout d'abord signaler qu'il ne se situe pas dans la zone classée par 1'UNESCO mais dans sa zone tampon, ensuite, il est au cœur d'un quartier qui va être prochainement transformé par la mise en place de l'Opération d'Intérêt National baptisée Bordeaux-Euratlantique, des frais seront alors engagés par la ville et les promoteurs privés dans ce secteur lors de ces travaux. Il y est prévu 15000 logements, $500000 \mathrm{~m}^{2}$ de bureaux ainsi que des commerces et des équipements publics. L'avenir nocturne des quais de Paludate est-il menacé, non par la gentrification, mais par la création d'un quartier d'affaire, ou au contraire, est-ce une formidable opportunité de créer le premier quartier de Bordeaux fonctionnant en continu ? Cela est envisageable mais avec une certaine nuance tout de même pour la direction générale de l'aménagement de la mairie de Bordeaux qui écrit dans l'ouvrage quartier[s]-Bordeaux sud: " [...] un pôle nuit [...] est compatible avec un futur quartier d'affaires et avec une grande gare...A condition que les nuisances soient contenues et que les lieux de la nuit soient à la fois branchés et irréprochables. " (2009). Les autorités sont donc favorables à un pôle nocturne, à condition qu'il corresponde à l'image qu'elles désirent. Tout ce qu'en disent les responsables du projet interrogés lors des concertations publiques, est qu'il y aura moins d'établissements.

\section{Conclusion :}

Bordeaux est une ville qui a beaucoup changé ces dernières années dans sa morphologie, sa sociologie et dans les pratiques quotidiennes de ses habitants, ce qui lui a permis de sortir de sa léthargie. Ces changements sont visibles de jour comme de nuit. Les lieux anciennement attractifs la nuit le sont toujours, c'est le cas de la Victoire et des quais de Paludate, certains le sont devenus plus récemment comme le quartier St-Pierre ou la promenade des quais du pont de pierre au bassin à flots. Les étudiant-e-s, comme de nombreux habitants et touristes, pratiquent ces lieux.

De manière générale, quel que soit le sexe des étudiants, leurs pratiques et leurs représentations sont proches. Certains aspects se trouvent malgré tout être caractéristiques du genre de l'étudiant, on pourrait presque dire stéréotypés: les étudiants sortent davantage pour faire des rencontres amoureuses ou boire de l'alcool, voire pour se saouler. Les étudiantes, quant à elles, se démarquent par le temps qu'elles consacrent aux préparatifs de sortie, à " se faire belle ", et par une plus grande crainte de la nuit, d'où une pratique territoriale différenciée et adaptée qui les conduit à sortir plus majoritairement à St-Pierre, considéré comme un quartier sûr depuis les rénovations qu'il a subi. Cette perception du danger est la différence la plus marquante et ayant une incidence importante sur les pratiques territoriales nocturnes des deux 
sexes. Ces résultats seraient certainement les mêmes pour une population non étudiante, comme le montre le travail de Marylène Lieber sur les femmes dans l'espace public.

Si la gentrification est la solution pour rendre la ville plus accessible, ne faut-il pas craindre ses conséquences bien connues que sont l'homogénéisation et les discriminations ?

\section{Bibliographie :}

- Bernié-Boissard, C. (2008). Des mots qui font la ville. Paris: La dispute.

- Bidou-Zachariasen C. (dir.) (2003). Retours en ville - des processus de "gentrification" urbaine aux politiques de "revitalisation" des centres, Paris, Descartes et Cie, collection "Les urbanités".

- $\quad$ Bureau, L. (1997). Géographie de la nuit. Montréal: L'hexagone.

- Delattre, S. (2000). Les douze heures noires. La nuit à Paris au XIXe siècle (2ème ed. 2003). Paris: Albin Michel.

- Deleuil, J.-M. (1994). Lyon la nuit. Lyon: Presses universitaires de Lyon.

- Dorthes, P. (2007). Bordeaux mode de ville. Bordeaux: pleine page.

- Espinasse, c. \& Buhagiar, P. (2004). Les passagers de la nuit, vie nocturne des jeunes. Paris: L'harmattan.

- Géronimi, M. (2008). La rue, la nuit à Montréal: paysages festifs et identitaires. In Sites publics, lieux communs (2). Urbanité et aménagement des rues et des ponts au Québec et au Canada (pp. 151-162). Pessac: MSHA.

- Godier, P., Sorbets, C., Tapie, G. (2009).Bordeaux métropole - Un futur sans rupture.

- Gwiazdzinski, L. (2005). La nuit, dernière frontière de la ville. La tour d'aigues: éditions de l'aube.

- Gwiazdzinski, L. (2007). Nuits d'Europe. Pour des villes accessibles et hospitalières. Belfort: Université de technologie de Belfort-Montbéliard (UTBM).

- Lieber, M. (2008). Genre, violences et espaces publics. La vulnérabilité des femmes en question. Paris : Presse de Sciences Po.

- Moutchouris A. (2003). Les jeunes de la nuit. Représentations sociales des conduites nocturnes. Paris : L'Harmattan.

- Paquot, T. (2006). Des corps urbains. Sensibilités entre béton et bitume. Paris: Autrement.

- Roulleau-Berger, L. (2004). La rue, miroir des peurs et des solidarités. Paris: PUF. 
- Victoire, E. (2007). Sociologie de Bordeaux. Paris: La Découverte

- Ville de Bordeaux, (2009). Portrait de quartier(s). 1. Bordeaux sud. Bordeaux : Éditions Overworld. 